\title{
Chiral Hadronic Mean Field Model including Quark Degrees of Freedom
}

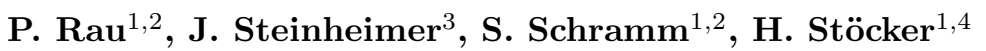 \\ ${ }^{1}$ Institut für Theoretische Physik, Goethe Universität, Max-von-Laue-Str. 1, \\ 60438 Frankfurt am Main, Germany \\ ${ }^{2}$ Frankfurt Institute for Advanced Studies (FIAS), Ruth-Moufang-Str. 1, 60438 \\ Frankfurt am Main, Germany \\ ${ }^{3}$ Lawrence Berkeley National Laboratory, Berkeley, CA 94720, USA \\ ${ }^{4}$ GSI Helmholtzzentrum für Schwerionenforschung GmbH, Planckstr. 1, 64291 \\ Darmstadt, Germany \\ E-mail: rau@th.physik.uni-frankfurt.de
}

\begin{abstract}
In an approach inspired by Polyakov loop extended NJL models, we present a nonlinear hadronic $\mathrm{SU}(3) \sigma-\omega$ mean field model augmented by quark degrees of freedom. By introducing the effective Polyakov loop related scalar field $\Phi$ and an associated effective potential, the model includes all known hadronic degrees of freedom at low temperatures and densities as well as a quark phase at high temperatures and densities. Hadrons in the model exhibit a finite volume in order to suppress baryons at high $T$ and $\mu$ This ensures that the right asymptotic degrees of freedom are attained for the description of strongly interacting matter and allows to study the QCD phase diagram in a wide range of temperatures and chemical potentials. Therefore, with this model it is possible to study the phase transition of chiral restoration and deconfinement. In this paper, the impact of quarks on the resulting phase diagram is shown. The results from the chiral model are compared to recent data from lattice QCD.

PACS numbers: 12.38.-t, 11.30.Rd, 14.20.Gk, 25.75.Nq
\end{abstract}

Submitted to: J. Phys. G: Nucl. Phys. 
Chiral Hadronic Mean Field Model including Quark Degrees of Freedom

\section{Introduction}

Deducing the properties of strongly interacting QCD matter at high temperatures and densities is one of the major goals of heavy ion collision experiments, as performed at the Relativistic Heavy Ion Collider (RHIC), the Large Hadron Collider (LHC), or at the upcoming Facility for Antiproton and Ion Research (FAIR). Experiments at the LHC and RHIC strongly suggest that at sufficiently large beam energies, i.e. high temperatures $T$ and small baryochemical potentials $\mu_{B}$, a new state of matter is created, comparable to a nearly perfect fluid with very low viscosity [1-4]. Possible phase transitions in other regions of the phase diagram with larger chemical potentials and baryonic densities are probed at lower beam energies.

Quantum chromodynamics (QCD) is the theoretical framework for the description of strongly interacting matter. A great difficulty in dealing with the QCD Lagrangian results from the fact that the equations of QCD cannot be solved analytically and perturbative approaches on QCD are suitable only in the high-temperature regime. Nevertheless, there is strong evidence for the existence of two phase transitions for strongly interacting matter. One is the chiral phase transition from a state with spontaneously broken chiral symmetry at low temperatures to a chirally symmetric phase with vanishing constituent quark masses [5 8]. For this transition, the chiral condensate $\sigma=\langle\bar{q} q\rangle$ serves as a well-defined order parameter. At vanishing baryochemical potentials $\mu=0$, lattice QCD methods can be used to study the chiral transition. In this theoretical approach, the QCD Lagrangian is treated numerically on a space-time grid. Calculations with different lattice actions consistently suggest a smooth cross-over transition with a "critical" temperature in the region of $T_{c} \approx$ $160 \mathrm{MeV}$ [ 18]. At non-zero chemical potentials, standard lattice QCD methods are not applicable due to the fermion sign problem. The fundamental phase structure of strongly interacting matter is still unknown. However, expansion and reweighting methods exist to extrapolate results from lattice QCD into the region $\mu>0$ of the phase diagram [10, 11, 19 28]. In this region, predictions on the order and exact location of the phase transition from different lattice groups are not consistent and depend on the used lattice action and other parameters such as the lattice spacing and the quark mass. While often the existence of a first-order phase transition and a critical endpoint at a certain chemical potential is predicted [12, 19, 29 31], other recent results suggest a smooth cross-over transition in $\sigma$ for the whole $T$ - $\mu$-plane under investigation [26, 32].

The other phase transition in QCD matter is the deconfinement transition from hadronic bound quark states to a quark-gluon plasma (QGP) state at high temperatures and baryonic densities [33] with the Polyakov loop $\Phi$ as a suitable order parameter.

Besides direct numerical approaches solving the QCD Lagrangian, a broad range of effective theoretical models exist for describing known properties of the phase diagram and thermodynamic characteristics of strongly interacting matter [5, 3440]. In this paper, such a model is presented including all known hadronic degrees of freedom [41 44] as well as a quark phase for the description of highly excited matter. With this model, it is possible to study the QCD phase diagram with a particular focus on the phase transitions. In the approach presented in the following, the description of the hadronic phase is done by a mean field sigma-omega type model and a quark phase is implemented following Polyakov-loop-extended Nambu-Jona-Lasinio (PNJL) models [45 55]. 
This paper is organized as follows. After this introduction, we present the quark hadron model and outline its theoretical background in Sec. 2. Results for the order parameters of chiral restoration and deconfinement phase transition and the thermodynamic properties of the model presented in Sec. 3. A short summary and concluding remarks are given in Sec. 4.

\section{Model approach}

For this study, we use a $S U(3)$-flavor $\sigma-\omega$ model with a non-linear realization of chiral symmetry; see Refs. [37, 41, 42, 56] for a more formal and detailed description of the original purely hadronic model. In this effective model, the Lagrangian in mean field approximation [35, 57] takes the form

$$
\mathcal{L}=\mathcal{L}_{\text {kin }}+\mathcal{L}_{\text {int }}+\mathcal{L}_{\text {meson }}
$$

where the first term represents the kinetic energy of the hadrons as described in Ref. 42] in more detail. The interaction of baryons and quarks with the scalar mesons fields $\sigma, \zeta$ (attractive interaction, see Eq. (3)) and the vector meson fields $\omega, \phi$ (repulsive interaction) respectively, is expressed by

$$
\mathcal{L}_{i n t}=-\sum_{i} \bar{\psi}_{i}\left[\gamma_{0}\left(g_{i \omega} \omega^{0}+g_{i \phi} \phi^{0}\right)+m_{i}^{*}\right] \psi_{i}
$$

The summation index $i$ runs over the three lightest quark flavors $(\mathrm{u}, \mathrm{d}, \mathrm{s})$, the baryonic octet, decuplet, and all heavier baryonic resonance states up to masses of $m_{N^{*}}=2.6 \mathrm{GeV}$, which have at least a three star rating in the the Particle Data Group listings [58, 59].

The mesonic condensates in our model are considered in mean field approximation. They include the scalar isoscalar field $\sigma \sim\langle\bar{u} u+\bar{d} d\rangle$ (which is identified as the chiral order parameter) and its strange equivalent $\zeta \sim\langle\bar{s} s\rangle$, as well as the vector isoscalars $\omega$ and $\phi$. The coupling strengths $g_{i \sigma, \zeta}$ of quarks and baryons to the scalar fields account for their effective mass, which to a large extent is generated dynamically

$$
m_{i}^{*}=g_{i \sigma} \sigma+g_{i \zeta} \zeta+\delta m_{i} .
$$

The explicit mass term $\delta m_{i}$ is chosen such as to reproduce the tabulated vacuum masses, which is the mean mass for broad resonance states. The lightest baryons exhibit an explicit mass of $\delta m_{i}=150 \mathrm{MeV}$ and somewhat larger values with increasing vacuum mass of the specific particle. The quark masses are set to $\delta m_{u, d}=6 \mathrm{MeV}$ and $\delta m_{s}=105 \mathrm{MeV}$. According to Eq. (3) the decrease of the $\sigma$-field at high temperatures and densities leads to decreasing baryon masses and thus to the restoration of chiral symmetry. The resulting effective masses and the connected ground state properties are discussed in detail at the beginning of Sec. 3 .

In the same manner, the effective chemical potential for quarks and baryons is given by

$$
\mu_{i}^{*}=\mu_{i}-g_{i \omega} \omega-g_{i \phi} \phi,
$$

and therefore, its value is generated by the couplings $g_{i \omega, \phi}$ of quarks and baryons to the vector meson fields.

The mesonic part of the Lagrangian Eq. (1)

$$
\mathcal{L}_{\text {meson }}=\mathcal{L}_{\text {vec }}+\mathcal{L}_{0}+\mathcal{L}_{\text {ESB }}
$$


includes the mass terms and the self interactions of the vector mesons

$$
\begin{aligned}
\mathcal{L}_{\mathrm{vec}}= & \frac{1}{2} \frac{\chi}{\chi_{0}}\left(m_{\omega}^{2} \omega^{2}+m_{\phi}^{2} \phi^{2}\right) \\
& +g_{4}\left(\omega^{4}+\frac{\phi^{4}}{4}+3 \omega^{2} \phi^{2}+\frac{4 \omega^{3} \phi}{\sqrt{2}}+\frac{2 \omega \phi^{3}}{\sqrt{2}}\right),
\end{aligned}
$$

as well as the self interaction terms of the scalar mesons explicitly given by the terms

$$
\begin{aligned}
\mathcal{L}_{0}= & -\frac{1}{2} k_{0}^{\prime}\left(\sigma^{2}+\zeta^{2}\right)+k_{1}\left(\sigma^{2}+\zeta^{2}\right)^{2} \\
& +k_{2}\left(\frac{\sigma^{4}}{2}+\zeta^{4}\right)+k_{3}^{\prime} \sigma^{2} \zeta \\
& -k_{4} \chi^{4}-\frac{1}{4} \chi^{4} \ln \frac{\chi^{4}}{\chi_{0}^{4}}+\frac{\delta}{3} \chi^{4} \ln \frac{\sigma^{2} \zeta}{\sigma_{0}^{2} \zeta_{0}}
\end{aligned}
$$

and an explicit symmetry breaking, defined by the terms

$$
\mathcal{L}_{\mathrm{ESB}}=-\frac{\chi^{2}}{\chi_{0}^{2}}\left[m_{\pi}^{2} f_{\pi} \sigma+\left(\sqrt{2} m_{k}^{2} f_{k}-\frac{1}{\sqrt{2}} m_{\pi}^{2} f_{\pi}\right) \zeta\right] .
$$

The scalar dilaton field $\chi$ can be identified as the gluon condensate. It was introduced in order to ensure scale invariance of the Lagrangian and to model QCD trace anomaly [42]. In the purely hadronic scenarios without considering a quark phase, changes in the $\chi$-field are very small and thus it is kept fixed at its ground state value $\chi_{0}$. This does not apply, in the presence of the quark phase. In this case, the quarks couple to the dilaton field [cf. Eq. [20] ] and hence, its value changes with the temperature. The chosen parameters of the Lagrangian are listed in Tab. 1.

The mesonic potential, which sets the dynamically generated meson masses, is defined as $\mathcal{V}_{\text {meson }}=-\mathcal{L}_{\text {meson }}$. From this, the masses of the mesons in the model are obtained by the second derivative of the potential with respect to the particular meson species $\xi_{j}$

$$
m_{j}^{* 2}=\frac{\partial^{2}}{\partial \xi_{j}^{2}} \mathcal{V}_{\text {meson }}
$$

In our model the coupling strengths of the baryons and the quarks to the fields are the parameters with the biggest impact on the resulting mater properties and the phase diagram. The couplings of the baryonic octet are fixed such as to reproduce the well-known vacuum masses and nuclear saturation properties (see Refs. 56, 60] for details). For the couplings of the baryonic resonances to the fields, a straight

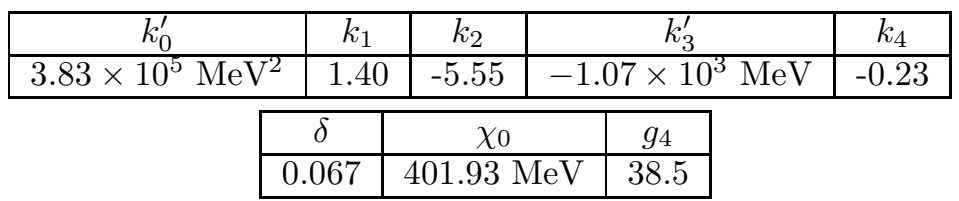

Table 1. General parameters used in the mesonic part of the chiral model Lagrangian Eq. (5). The couplings of the particles to the meson fields are given in Tab. 2 


\begin{tabular}{|c|c|cc|cc|}
\hline & $\delta m_{0}[\mathrm{MeV}]$ & $g_{\sigma}$ & $g_{\zeta}$ & $g_{\omega}$ & $g_{\phi}$ \\
\hline \hline $\mathrm{u}$ & 6.0 & -3.5 & 0.0 & 4.0 & 0.0 \\
$\mathrm{~d}$ & 6.0 & -3.5 & 0.0 & 4.0 & 0.0 \\
$\mathrm{~s}$ & 105.0 & 0.0 & -3.5 & 0.0 & 7.1 \\
\hline $\mathrm{N}$ & 150 & -9.83 & 1.22 & 11.56 & 0.0 \\
\hline
\end{tabular}

Table 2. Explicit particle masses $\delta m_{0}$ and coupling strengths of the quarks and the nucleons $g_{i}$ to the fields. In the non-interacting HRG scenario, all couplings are set to zero (cf. Fig. 5 for detailed parameter study).

forward two parameter ansatz is chosen [43]. This approach avoids having multiple different coupling parameters for every single resonance state or $\mathrm{SU}(3)$ multiplets. The resonance couplings are connected to those of the nucleons to the respective fields via the relations

$$
\begin{aligned}
g_{B_{i} \sigma, \zeta} & =r_{s} g_{N \sigma, \zeta}, \\
g_{B_{i} \omega, \phi} & =r_{v} g_{N \omega, \phi},
\end{aligned}
$$

where $r_{s}$ and $r_{v}$ are the so-called scaling parameters for the scalar and vector fields. Ref. [43] demonstrates the impact of baryonic resonance states on the phase diagram. This was essentially done by varying the vector coupling strength (change the value of $r_{v}$, which effectively controls the abundance of particles at finite baryochemical potentials in the model). For reasonable couplings, a first-order phase transition ceases to exist in favor of a smooth cross-over due to the continuous population of heavier resonances states.

In this study, the vector coupling parameter is kept fixed at $r_{v}=0.9$. Furthermore, in order to ensure a smooth cross-over of the order parameter and of the thermodynamic quantities at vanishing chemical potentials, the scalar couplings are also fixed at a value close to unity $r_{s} \approx 1$. For the non-interacting hadron resonance gas (HRG), the couplings of all particles to the fields are set to zero and $\delta m_{i}$ is kept fixed at the respective vacuum mass values [58, 59].

The couplings of the quarks to the meson fields are controlled by $g_{q \sigma}, g_{q \zeta}, g_{q \omega}$, and $g_{q \phi}$, where $q$ stands for the respective quark species (u, d, s). The explicit masses of the quarks and the nucleons $\delta m_{0}$ and their coupling strengths to the fields are listed in Tab. 2. In Sec. 3.1] a detailed study of the impact of the quark couplings on the phase diagram and the nuclear ground state is performed.

All thermodynamic quantities in our model can be derived from the grand canonical potential, which is defined as

$$
\frac{\Omega}{V}=-\mathcal{L}_{\text {int }}-\mathcal{L}_{\text {meson }}+\frac{\Omega_{\mathrm{th}}}{V}-U_{\mathrm{Pol}} .
$$

In the heat bath of hadrons and quarks, the thermal contribution to the potential includes those from quarks, baryons, and mesons

$$
\Omega_{\mathrm{th}}=\Omega_{\mathrm{q} \overline{\mathrm{q}}}+\Omega_{\mathrm{B} \overline{\mathrm{B}}}+\Omega_{\mathrm{M}} .
$$


In explicit form, the thermal energy contributions are defined as

$$
\begin{aligned}
\Omega_{\mathrm{q} \overline{\mathrm{q}}}= & -T \sum_{i \in q} \frac{\gamma_{i}}{(2 \pi)^{3}} \int d^{3} k\left(\ln \left[1+\Phi e^{-\frac{1}{T}\left(E_{i}^{*}(k)-\mu_{i}^{*}\right)}\right]\right. \\
& \left.+\ln \left[1+\bar{\Phi} e^{-\frac{1}{T}\left(E_{i}^{*}(k)+\mu_{i}^{*}\right)}\right]\right)
\end{aligned}
$$

for the quarks and antiquarks $(i \in\{u, d, s\}$ ), which couple to the effective Polyakov loop potential $\Phi$ and its conjugate $\bar{\Phi}$ as described below,

$$
\begin{gathered}
\Omega_{\mathrm{B} \overline{\mathrm{B}}}=-T \sum_{j \in B} \frac{\gamma_{j}}{(2 \pi)^{3}} \int d^{3} k\left(\ln \left[1+e^{-\frac{1}{T}\left(E_{j}^{*}(k)-\mu_{j}^{*}\right)}\right]\right. \\
\left.+\ln \left[1+e^{-\frac{1}{T}\left(E_{j}^{*}(k)+\mu_{j}^{*}\right)}\right]\right),
\end{gathered}
$$

for baryons and antibaryons with the sum running over all baryons $B$, and

$$
\Omega_{\mathrm{M}}=T \sum_{l \in M} \frac{\gamma_{l}}{(2 \pi)^{3}} \int d^{3} k \ln \left[1-e^{-\frac{1}{T}\left(E_{l}^{*}(k)-\mu_{l}\right)}\right] .
$$

for the mesons, where in this case the sum runs over all mesons. The spin-isospindegeneracy factor is denoted with $\gamma_{i, j, l}$ for the respective particle species $i, j, l$ and $E_{i, j, l}^{*}(k)=\sqrt{k^{2}+m_{i, j, l}^{* 2}}$ for the single particle energies.

The introduction of the quarks in our model is done as in other recent extensions of the original Nambu-Jona-Lasinio models 5, 50] which include an effective Polyakov loop potential. The Refs. [45, 47, 48, 53, 61 63] give a comprehensive review of these PNJL models.

The scalar Polyakov loop field $\Phi$ in Eq. (14) is retrieved by tracing the constant matrix valued temporal component of the $\mathrm{SU}(3)$ color gauge background field

$$
\Phi=\frac{1}{3} \operatorname{Tr}\left(e^{-A_{0} / T}\right) .
$$

Phenomenologically, this field indicates the phase transition from a confined hadronic phase to a deconfined partonic phase and therefore from the hadron resonance gas to the quark phase. These dynamics are controlled by the effective Polyakov loop potential, adopted from Ref. [63], in the form

$$
\begin{array}{r}
U=-\frac{1}{2} a(T) \bar{\Phi} \Phi+b\left(\frac{T_{0}}{T}\right)^{3} \ln [1-6 \bar{\Phi} \Phi \\
\left.+4\left(\bar{\Phi}^{3}+\Phi^{3}\right)-3(\bar{\Phi} \Phi)^{2}\right]
\end{array}
$$

which fulfills the $\mathrm{Z}(3)$ center symmetry of pure gauge QCD. The temperature dependent parameter

$$
a(T)=a_{0}+a_{1}\left(\frac{T_{0}}{T}\right)+a_{2}\left(\frac{T_{0}}{T}\right)^{2}
$$

and the parameters therein are chosen such as to reproduce lattice data for QCD thermodynamics in the pure gauge sector [64] as well as known features of the 


\begin{tabular}{|c|c|c|c|c|}
\hline$T_{0}[\mathrm{MeV}]$ & $a_{0}$ & $a_{1}$ & $a_{2}$ & $b$ \\
\hline $225-235$ & 3.51 & -2.47 & 15.2 & -1.75 \\
\hline
\end{tabular}

Table 3. Parameters of the effective Polyakov loop potential Eqs. (18), (19) and coupling parameters of the model. The value of $T_{0}$ is adapted according to couplings (see text).

deconfinement transition [63]. By doing so, the potential reproduces the pressure, the energy density, and the entropy density in the pure gluonic sector given by lattice QCD calculations. At low temperatures, in the confined phase, $\Phi$ is zero and its value rises with increasing temperatures. It tends to values $\Phi \rightarrow 1$ above the critical Polyakov temperature $T_{0}$ and with increasing chemical potentials. Generally, in PNJL models, the gluon dynamics are reduced to the static background field as given by the Polyakov loop and to point couplings. As C. Ratti et al. point out in Ref. [47], due to these simplifications of the gluon dynamics and the insufficient treatment of transverse gluons, which are dominant degrees of freedom at very high temperatures, the PNJL model is likely to deviate from lattice QCD thermodynamics in the high-temperature region (see also [65]).

The model parameters for the Polyakov loop potential are listed in Tab. 3. For the free parameter of the Polyakov loop $T_{0}$, a value of $235 \mathrm{MeV}$ is chosen when used in the non-interacting scenario, in which all couplings are set to zero, and $225 \mathrm{MeV}$, when all particles in the model couple to the respective meson fields as described above.

Since the frozen dilaton field $\chi=\chi_{0}$ constrains the chiral condensate to finite values for all $T$ and $\mu$, a coupling of the Polyakov loop potential to the dilaton field is introduced. This suppresses $\sigma$ when deconfinement is achieved. It is realized via

$$
\chi=\chi_{0}\left[1-\left(\frac{\Phi^{2}}{2}+\frac{\bar{\Phi}^{2}}{2}\right)^{2}\right],
$$

a form similar to the one described in Ref. [66]. This coupling leads to a stronger and more reasonable suppression of the chiral condensate in the presence of free quarks in the last three terms of the scalar meson self interaction in Eq. (77).

Minimizing the grand canonical potential Eq. (12) for a given temperature and baryochemical potential with respect to its respective fields, leads to a coupled system of non-linear equations of motion for the fields and the particle densities $\rho_{i, j, l}$. In the following, all thermodynamic quantities are derived starting with the pressure $p=-\partial \Omega / \partial V$ and the entropy density $s=\partial p / \partial T$ by using the expression for the internal energy $\epsilon=T s-p V+\sum_{i} \mu_{i} \rho_{i}$, with the summation index $i$ running over all particle species in the model.

In order to use hadronic degrees of freedom at low temperatures and densities and quarks at higher temperatures and densities, we introduce an eigenvolume $V_{\mathrm{ex}}^{i}$ for each particle species $i$, as it was done in Refs. [55, 67, 68]; similar approaches to repulsive volume interactions are carried out in Refs. 69 73. By doing so, it is ensured that with increasing densities at higher $T$ and $\mu$ - at the latest when quark abundances rise quickly at the deconfinement phase transition - an effective suppression of hadronic degrees of freedom is established due to excluded volume effects, as was shown in Refs. 74 77]. An eigenvolume for every particle of a species $i$ is introduced and expressed by

$$
V_{\mathrm{ex}}^{i}=\frac{1}{2} \frac{4}{3} \pi(2 r)^{3}
$$


with the parameter $r$ defining the effective radius of the hadrons in the model. For the relation between the baryon and the meson radii, we assume $r_{M}=r_{B} / 2$. For calculations including the quark phase and assuming full couplings to the meson fields, a value $V_{\mathrm{ex}}^{B}=2.7 \mathrm{fm}$ for the baryons and $V_{\mathrm{ex}}^{B} / 8$ for the mesons is chosen (cf. Tab. (4). Quarks do not exhibit a finite volume.

In this way, each single hadron occupies a certain volume and thereby reduces the total volume $V$ by $V_{\mathrm{ex}}^{i}$. This results in a modified chemical potential

$$
\mu_{j}^{*}=\mu_{j}-\sum_{i} V_{\mathrm{ex}}^{i} p^{i}
$$

where $p^{i}$ denotes the partial pressure of species $i$.

The particle, energy, and entropy densities are corrected by the excluded volume and found to be

$$
\rho_{i}^{\prime}=\rho_{i} \xi_{i}^{V}, \quad e^{\prime}=\sum_{i} e_{i} \xi_{i}^{V}, \quad s^{\prime}=\sum_{i} s_{i} \xi_{i}^{V},
$$

with the correction factor given as $\xi_{i}^{V}=1 /\left(1+V_{\mathrm{ex}}^{i} \rho_{i}\right)$. This definition of $\mu_{j}^{*}$ and the volume corrected thermodynamic quantities ensures that thermodynamic consistency holds true in the whole $T$ - $\mu$-plane. That means, the relation for the internal energy, as given above, and all partial derivatives for determining the thermodynamic quantities remain valid.

\section{Results}

\subsection{Model properties}

First, we present results from the described model at vanishing baryochemical potential, $\mu_{B}=0$. Figure 1 shows the order parameter for the chiral phase transition normalized to its ground state value $\sigma / \sigma_{0}$ as a function of the temperature. The order parameter for the deconfinement transition $\Phi$ as a function $T$ is shown in Fig. 2 The results from our model are compared to lattice QCD results (data points or gray band) using different improved staggered fermion actions. In detail, these are the asqtad action from Ref. 78], the HISQ action from Ref. 79, 80, 86], the p4 action from Ref. [78, 81, 82], and the stout action from Ref. [83 85]. For the calculations with the described chiral model, here and in the following, four different scenarios, i.e. basic parameter sets with the following abbreviations, are distinguished:

HRG describes the pure hadron resonance gas without excluded volume suppression and a quark phase that does not interact with the mesonic fields.

Int. HRG same as above with the only difference, that all hadrons couple to the meson fields as described above.

$\mathbf{H R G}+\mathbf{q}$ includes the hadrons with excluded volume effects and a quark phase. All couplings to the mesonic field are set to zero only the quarks couple to the Polyakov loop.

Int. HRG $+\mathbf{q}$. Same as above but here, all couplings to the meson fields are set to the finite values given in Tab. 2 ,

A summarizing table of the configurations for these scenarios is given in Tab. (4) For the full interacting scenario, a value $V_{\mathrm{ex}}=2.7 \mathrm{fm}^{3}$ is chosen for the baryons. This 


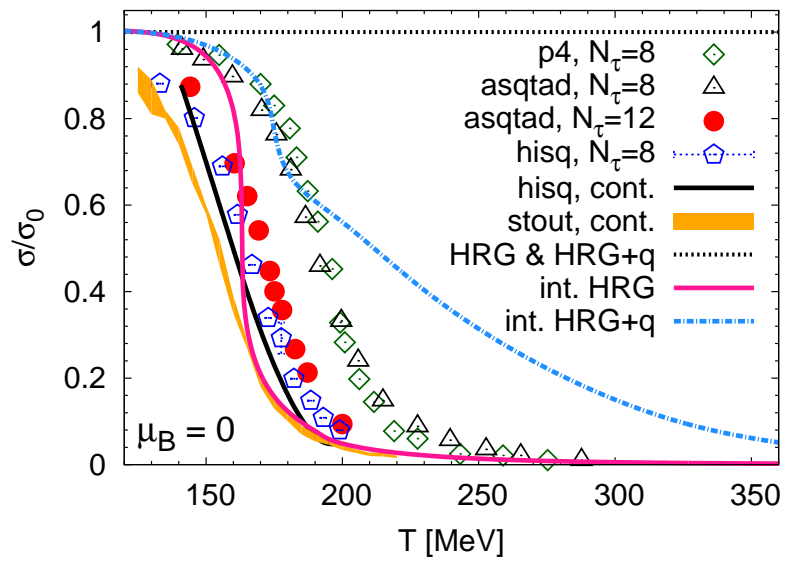

Figure 1. (Color online) Normalized order parameter for the chiral condensate $\sigma / \sigma_{0}$ as a function of the temperature compared to lattice data from Refs. 7885]. The red solid line depicts the results from the model for the interacting hadron resonance gas (HRG) without a quark phase. The interacting HRG with a quark phase is shown by the blue dashed line and the green dashed line shows the results for hadrons that do not interact with the mesons fields, together with the described quark phase. Comparing the curves, it clearly shows that even when including quarks, the chiral phase transition is mainly driven by hadronic degrees of freedom.

\begin{tabular}{|c|c|c|c|c|c|}
\hline & $V_{\text {ex }}$ & $g_{\text {Bs }}$ & $g_{\text {Bv }}$ & $g_{\text {qs }}$ & $g_{\text {qv }}$ \\
\hline HRG & 0.0 & X & X & & \\
int. HRG & 0.0 & $\checkmark$ & $\checkmark$ & & \\
\hline HRG+q & 1.8 & X & X & X & X \\
int. HRG+q & 2.7 & $\checkmark$ & $\checkmark$ & $\checkmark$ & $\checkmark$ \\
\hline
\end{tabular}

Table 4. The four different scenarios considered here include the non-interacting HRG and the interacting HRG, both with and without a quark phase q. For all scenarios, the values for the excluded volume $V_{\text {ex }}$ are given in $\mathrm{fm}^{3}$. The couplings of the baryons $B$ and the quarks $q$ to the scalar and the vector meson fields $\left(g_{\mathrm{Bs}, \mathrm{v}}\right.$, $\left.g_{\mathrm{qs}, \mathrm{v}}\right)$ are either set to zero when marked with a cross, or have the finite value listed in Tab. 2 if indicated by a check mark (see text). The first two scenarios are purely hadronic and do not include a quark phase.

corresponds to a mean baryon radius of $r_{B}=0.86 \mathrm{fm}$ which is slightly smaller than the measured proton charge radius $r_{p}=0.88 \mathrm{fm}$ [87].

The results for the purely hadronic resonance gas that is interacting with the meson fields as described above (int. HRG) is depicted by the solid magenta line (color online). In this case, at the critical temperature $T_{c}=164 \mathrm{MeV}$, which quantitatively agrees well with recent lattice data, $\sigma$ falls off in a small temperature range. As already pointed out in Ref. [43], a slower decrease of $\sigma$, as it is observed in the lattice data, can be achieved by taking into account pionic self interactions [88 90]. Nevertheless, as predicted by all lattice QCD calculations, the transition is a smooth cross-over for both order parameters. As found in Ref. [43], the inclusion of all known hadronic degrees of freedom in a $\sigma-\omega$ model restricts the chiral phase transition to a smooth cross-over in the whole $T$ - $\mu$ plane. Nevertheless, the first-order liquid-gas phase transition for nuclear matter at the ground state up to a critical end-point $T_{c} \approx 15 \mathrm{MeV}$ stays 


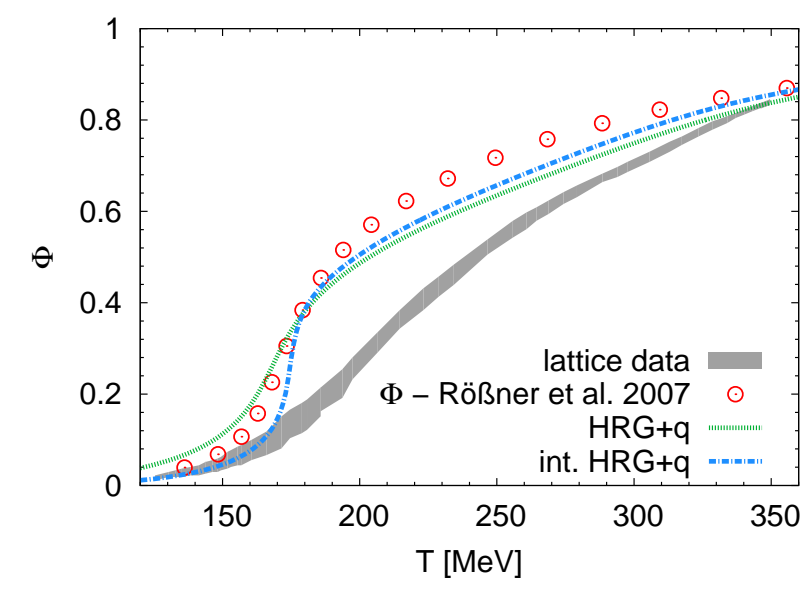

Figure 2. (Color online) The Polyakov loop order parameter $\Phi$ for the deconfinement phase transition as a function of $T$ at $\mu=0$. For the two scenarios including the quark phase, the non-interacting scenario (green line) and the interacting scenario (blue line), $\Phi$ only shows rather small differences below the critical temperature $T_{c} \approx 170 \mathrm{MeV}$. Our results are close to those from Ref. [48] (red points) from which the Polyakov loop potential was adopted. Other parameterizations of the Polyakov loop potential (e.g. given in Ref. 46]) yield a very similar slope of $\phi$. The gray band covers the lattice QCD results for $\Phi$ given in Refs. 78 85].

present in our model.

The second scenario considered here, is the interacting HRG with the described PNJL-like quark phase (blue dashed line). The decrease of $\sigma$ is also very strong but the slope flattens significantly at the critical temperature for the Polyakov loop $T_{c}=175 \mathrm{MeV}$ as shown in Fig. 2. However, in comparison to the pure HRG it shows, that the slope of $\sigma(T)$ does not change up to $T_{c}$ and therefore that the chiral phase transition is mainly driven by hadronic degrees of freedom.

Since quarks in the model do not have an eigenvolume, quark degrees of freedom are strongly preferred to be occupied at $T_{c}$ and above. As of this temperature, the number of quarks exceeds the number of baryons and mesons in the systems. Their smaller coupling strength to the chiral condensate (cf. Tab. 2) leads to a much slower decrease in $\sigma$. On the other hand, the rapid increase of the quark multiplicities at $T_{c}$ generates a distinctly steeper slope of the Polyakov loop parameter $\Phi$ (b) than predicted by lattice QCD data, which is compiled in the gray band in Fig. 2 ,

In contrast to this finding, the Polyakov loop $\Phi$ results reproduce those from Ref. [48] depicted by the red circles. More recent PNJL models [46] also show a significant overshooting of the lattice results in the transition region revealing an obvious discrepancy between effective models and first-principle calculations on this point. In lattice QCD, there is no sharp increase in the deconfinement order parameter at $T_{c}$.

As two limiting cases, the order parameters for the non-interacting HRG is shown with [green dashed line in Fig. 2] and without a quark phase. The $\sigma$-parameter for the latter two scenarios are shown by the flat line at $\sigma / \sigma_{0}=1$. The excluded volume parameters of the baryons $V_{\text {ex }}$ for the four different scenarios considered had to be adapted in order to both give a smooth transition between the two phases and 


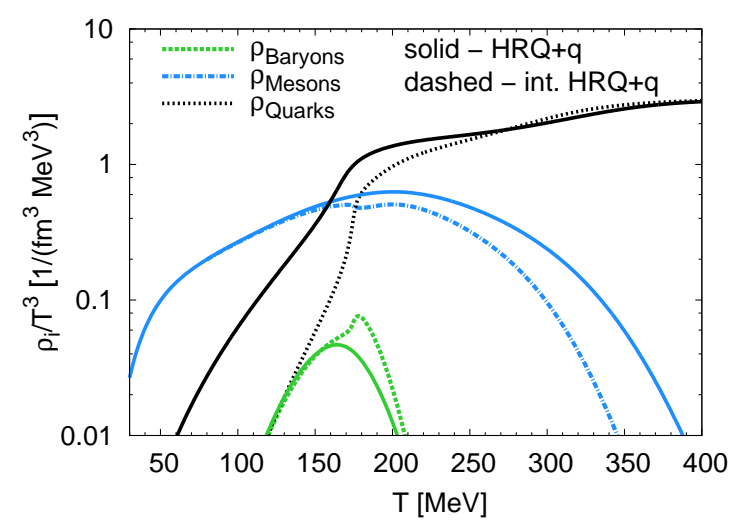

Figure 3. (Color online) Net baryon number densities over the temperature cubed $\rho_{i} / T^{3}$ of all baryons (green lines), mesons (blue lines) and quarks (black lines) as a function of the temperature at $\mu_{B}=0$. Solid lines depict results for the ideal HRG and dashed lines the interacting HRG both including the quark phase. For the eigenvolume of the baryons, $V_{\mathrm{ex}}=2.7 \mathrm{fm}^{3}$ is chosen for the interacting case and $V_{\mathrm{ex}}=1.8 \mathrm{fm}^{3}$ for the non-interacting HRG (see text). As expected, since there is no repulsive vector interaction for the quarks in the ideal scenario, the quark density below $T_{c}$ is much higher than in the fully interacting scenario.

a reasonable agreement with lattice data for the thermodynamic quantities. These requirements cause a rather large eigenvolume of the baryons in the fully interacting scenario.

The values chosen for the different model scenarios considered in this study are listed in Tab. 4. In the two scenarios with only a hadronic phase, on the other hand, hadrons do not carry an explicit eigenvolume and thus excluded volume corrections do not apply.

Figure 3 shows the total baryon number densities of baryons and antibaryons (green lines), mesons (blue lines), and quarks plus antiquarks (black lines) at $\mu=0$ as a function of the temperature, for the two scenarios containing a quark phase. The results are shown for the non-interacting ideal gas scenario (solid lines) and the fully interacting scenario with the non-zero couplings to the fields (dashed lines). Obviously, the coupling of the particles to the scalar fields, which generates the effective masses as defined by Eq. (3), leads to an increase in the particle multiplicities at $T_{c}$, in particular of the baryon multiplicity which, however, drops again shortly due to the large baryon volume in favor of a quickly rising quark multiplicity. In the temperature range $0.6 T_{c}<T<1.3 T_{c}$, there is a phase in which quarks and hadrons coexist, as expected for a cross-over transition. At a temperature $T \approx 1.3 T_{c}$, baryons vanish from the system.

In contrast, the quarks, show a stronger dependence on their couplings to the repulsive vector fields. If the quarks do not couple to the repulsive vector field, showing an unphysical behavior they appear even at temperatures far below $T_{c}$ and their total density is much larger than the baryon density for all temperatures. Obviously, this behavior has a strong impact on the nuclear ground state what will be studied below. If the mesons couple to the fields, the effective masses of the pseudoscalar mesons scale as $m_{\mathrm{mes}}^{* 2} \sim 1 / \sigma$ [43]. For this reason, at temperatures $T>T_{c}$ their multiplicities decrease significantly faster than in the non-interacting case due to an increasing mass. 

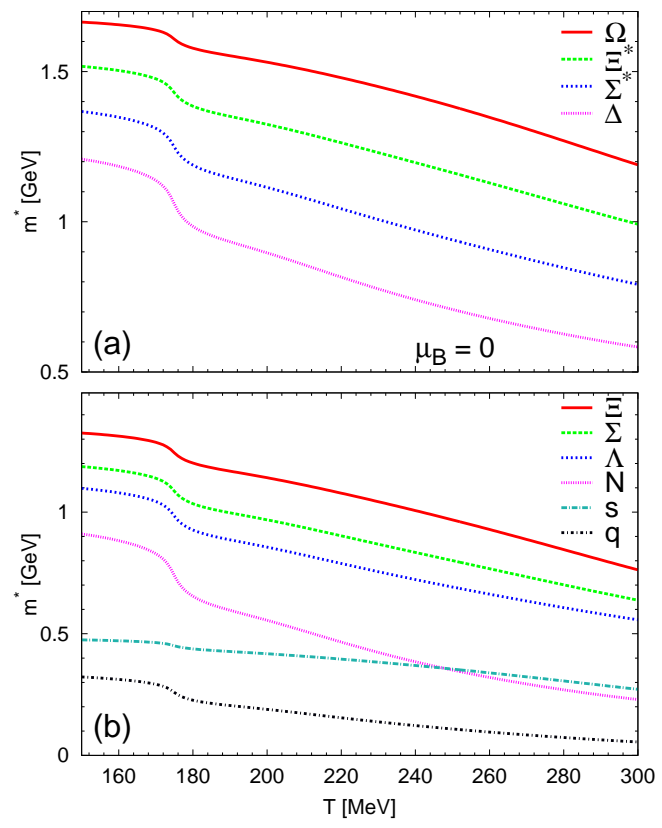

Figure 4. (Color online) Effective masses $m^{*}$ of the quarks ( $\mathrm{u}, \mathrm{d}=\mathrm{q}$ and $\mathrm{s}$ ), the baryonic octet (lower panel), and the decuplet (upper panel) for the fully interacting scenario including hadrons and quarks as a function of the temperature at $\mu_{B}=0$. All shown particles couple to the fields with coupling strengths as listed in Tab. 2 The change of the effective masses largely reflects the slope of the scalar $\sigma$-field (cf. blue line in Fig. 1); therefore the values for $m^{*}$ show a rather smooth decrease with increasing temperature.

This finding of hadrons even at temperatures above $T_{c}$, agrees well with considerations that light and strange resonances can exist in this region and are likely to be produced even in the QGP due to their short formation times [91, 92]. In Ref. [93] it was shown by comparing PNJL and lattice QCD calculations that hadronic bound states are expected to exist up to temperatures of $2 T_{c}$.

As defined by Eq. (33), the effective masses $m^{*}$ of the particles in the chiral model are dynamically generated by the coupling to the scalar meson fields. Increasing temperature and density decreases $\sigma$ and $m^{*}$ accordingly. In consequence, chiral symmetry can be restored. Figure 4 shows the effective masses of the baryons and of the quarks in the fully interacting scenario as a function of the temperature. The lower panel (b) shows the effective masses of the baryon octet $(N, \Lambda, \Sigma, \Xi)$, the up and down valence quarks, denoted as $q$, and the strange quark s. In the upper panel (a) the effective masses of the lowest lying baryon resonances in the decuplet $\left(\Delta, \Sigma^{*}\right.$, $\Xi^{*}, \Omega$ ) are shown. Since the decrease of the scalar fields happens rather smoothly with increasing temperature, the decrease of $m^{*}$ is also relatively slow. Nevertheless the steepest decline is still visible at $T_{c}$ 8, 94 . For nucleons, the mass at $T=1.5 T_{c}$ has dropped down to $0.33 m_{0}^{N}$, for the constituent up and down quarks to $0.27 m_{0}^{u, d}$ , and to $0.69 m_{0}^{s}$ for the strange quarks. Most baryons vanish at $T=1.3 T_{c}$. For this reason, the mean field approximation should not be affected by too small baryonic masses and possible fluctuations as argued in Refs. [95, 96]. 

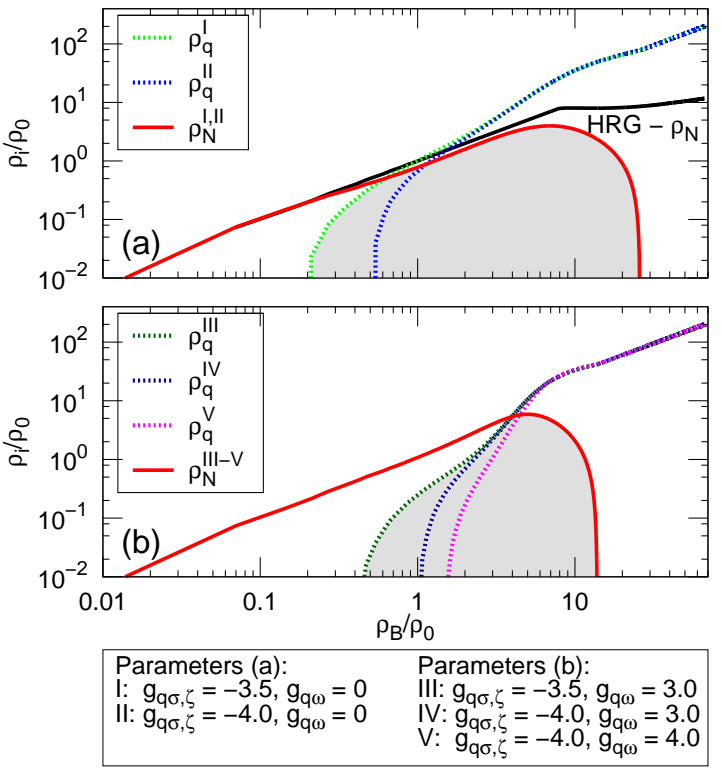

Figure 5. (Color online) Particle vector densities of up and down quarks $\rho_{q}$ (dashed lines) and of nucleons $\rho_{N}$ (solid red line) at $T=0$ as a function of $\rho_{B} / \rho_{0}$ for zero (a) and non-zero quark vector couplings $g_{q \omega}$ (b) and different values of the scalar couplings $g_{q \sigma, \zeta}$ (see parameter list below the plots). The gray shaded areas indicate possible coexistence of quarks and nucleons. If the quark vector couplings take a finite value (b), quarks are predominantly present at $\rho_{B}=4 \rho_{0}$ and all nucleons vanish within the model at $\rho_{B}=15 \rho_{0}$. The solid black line in (a) depicts $\rho_{N}$ for the purely hadronic model without quarks. To ensure that no free quarks are present in the ground state, both couplings have to be chosen sufficiently large.

In the hadronic version of this effective model, i.e. without the quark phase and excluded volume correction terms [41, 42], the couplings of the baryon octet to the mesonic fields and the mesonic potential were chosen in such a way as to reproduce the well-known vacuum masses of the baryons and the experimentally determined masses of the mesons, as well as the nuclear ground state properties (e.g. the correct binding energy per nucleon and the nuclear compression modulus), and the asymmetry energy. The implementation of the quark phase is done in a way which ensures that these requirements are still fulfilled. Moreover, one has to consider whether quarks already appear in the nuclear ground state.

First, we consider temperature zero. Figure 5 shows the vector particle densities of up and down quarks

$$
\rho_{q}=\sum_{i=u, d} \rho_{q_{i}}^{+}-\rho_{\bar{q}_{i}}^{-}
$$

together with the density of the nucleons $\rho_{N}$ at $T=0$, where the anti-particle density $\rho_{j}^{-}$is zero, as a function of the baryonic density $\rho_{B}$ for different sets of the quark coupling parameters (I-V, see key in figure) in the fully interacting model including hadrons and quarks. All densities are given in units of the ground state density $\rho_{0}$. In panel (a) the densities for a vanishing coupling of the quarks to the vector meson fields $g_{q \omega}=0$ and for two different values for the quark - scalar meson field coupling $\left(g_{q \sigma, \zeta}=-3.5\right.$, blue doted line, and $g_{q \sigma, \zeta}=-4.0$, magenta dashed line) are shown. It is 
obvious that without the coupling to the repulsive vector field, in both cases the quarks appear at densities smaller than the nuclear ground state density at $\rho_{B}=0.15 \mathrm{fm}^{3}$. This specific choice of quark coupling parameters may thus only be physically correct if the quarks in this region of the phase diagram are not considered to be unbound particles. The green dashed line in both panels of the figure shows the density of the nucleons $\rho_{N}$ which differs only slightly for the different parameter sets in (a) and (b). As a reference, the solid black line in panel (a) depicts the nucleon density in the purely hadronic model without a quark phase.

The values for the quark scalar coupling in panel (b) are the same as before and the value of the up and down quark vector coupling is varied between $g_{q \omega}=3.0$ and $g_{q \omega}=4.0$. It shows, that with a scalar coupling of $g_{q \sigma}=-4.0$ for the up and down quarks as well as $g_{q \zeta}=-4.0$ for the strange quarks and a vector coupling equal or larger than $g_{q \omega}=3.0$ for up and down quarks, all quarks are effectively suppressed below the nuclear ground state density as expected. Due to this finding, with the given choice of parameters, which is compatible with the corresponding parameters in the baryon sector, the quarks do not affect the nuclear ground state. In the case of non-zero quark vector couplings (b), quarks are predominantly abundant at $\rho_{B}=4 \rho_{0}$ and all nucleons within the model cease to exist at $\rho_{B}=15 \rho_{0}$.

With this parameter set, the model reproduces the nuclear saturation density $\rho_{0} \approx 0.15 \mathrm{fm}^{3}$ and the correct binding energy per nucleon $E / A\left(\rho_{0}\right) \approx-16 \mathrm{MeV}$. At the saturation density the compression modulus of infinite nuclear matter is given by [97]

$$
K_{\infty}=\left.k_{F}^{2} \frac{d^{2} E / A}{d k_{F}^{2}}\right|_{k_{F}=k_{F_{0}}}=\left.9 \rho_{0}^{2} \frac{d^{2} E / A}{d \rho^{2}}\right|_{\rho=\rho_{0}} .
$$

Calculations of isoscalar giant monopole and dipole resonances, i.e. compression modes of nuclei, and related experimental data suggest a value of $K_{\infty}=240 \pm 20 \mathrm{MeV}$ for the compression modulus of nuclear matter (see Ref. [98] for a recent comprehensive review on how to determine $K_{\infty}$ ). The purely hadronic model neglecting the eigenvolume of the particles, gives a value of $K_{\infty}=225 \mathrm{MeV}$. However, if quarks are included and the excluded volume effects are taken into account in order to suppress hadronic degrees of freedom above $T_{c}$, the pressure and $E / A$ as a function of the baryon density increases much faster depending on the choice of $V_{\mathrm{ex}}$. This leads to a significantly higher compressibility modulus, which is $K_{\infty}=370 \mathrm{MeV}$ for an eigenvolume of the baryons of $V_{\mathrm{ex}}=1 \mathrm{fm}^{3}$ and an unreasonably high value of $K_{\infty}=890 \mathrm{MeV}$ if $V_{\mathrm{ex}}$ is set to $2 \mathrm{fm}^{3}$.

This rapid increase in the density could be overcome by making the eigenvolume of the particles temperature or density dependent. Phenomenologically, it seems plausible that the eigenvolume of one hadron is not perceived by another particle further away at low temperatures and densities below the nuclear ground state, where particles are statistically distributed relatively far from each other in a large volume. For this reason, the eigenvolume effect would vanish in this region and it would reach its actual full value at higher densities, at which the particles in the system are densely packed and would start to overlap. This, on the one hand, would still result in the same effective suppression of hadrons at high temperatures and densities in favor for point-like quarks as before. On the other hand, the introduction of a density or temperature dependent eigenvolume needs to be done with care, as not to violate the thermodynamic consistency of the approach (cf. Eq. (23)) 73, 99, 100]. Particularly, since the transition between the hadronic and the quark phase is abrupt in 


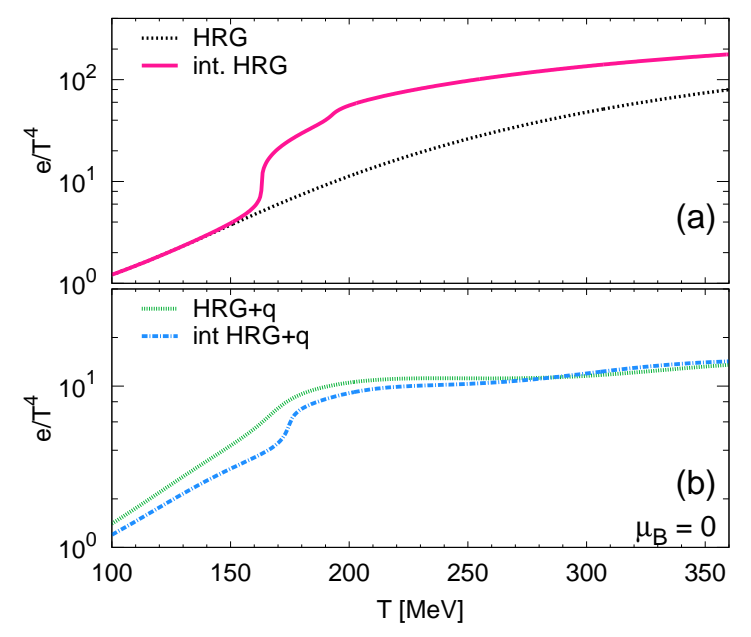

Figure 6. (Color online) Energy density over temperature to the fourth power at zero baryochemical potential from the presented model. Panel (a) shows results from the model without quarks. While the non-interacting HRG (black dashed line) rises monotonously, in the interacting scenario (solid magenta line) $e / T^{4}$ rises quickly at $T_{c}$ due to the drop in the hadron masses. Panel (b) shows the same quantity for the model including a quark phase. In the case without the meson field interactions (HRG $+\mathrm{q}-$ green line), $e / T^{4}$ grows much faster as in the interacting case (HRG $+\mathrm{q}$ - blue line), due to the non-existent vector repulsion of quarks. Unlike in a Hagedorn resonance gas, all quantities in the model saturate at very large temperatures caused by a large but still limited number of degrees of freedom.

the fully interacting model, this seems reasonable in order to achieve a much smoother transition. Work along this line is in progress.

\subsection{Thermodynamics}

We will now take a closer look at the thermodynamic properties of the model. Figure 6 shows the energy density divided by the temperature to the fourth power, $e / T^{4}$. Panel (a) depicts the purely hadronic model without quarks in the non-interacting scenario (black dashed line) as well as results from the scenario in which hadrons fully interact with the meson fields (solid magenta line). The interacting HRG shows a rapid rise of the energy at $T_{c}$. This is due to the drop in the scalar condensate $\sigma$. Therefore, also the effective hadron masses decrease rapidly, which leads to large hadron abundances in the absence of any volume based particle repulsion. The increase of the thermodynamic quantities of the ideal HRG is obviously much slower above $T_{c}$ since the masses are not dynamically generated via couplings to the scalar meson fields. The purely hadronic model is considered only to be valid at least up to temperatures in the region of $T_{c}$.

Panel (b) shows the results from the chiral model including the additional quark phase. Below the critical temperature, the energy density in the non-interacting scenario (green dashed line) is significantly higher than in the non-interacting scenario (blue dashed line). This is because in this case, there is no suppression by the vector meson fields and quarks are not suppressed at low temperatures (cf. Fig. (3)) Furthermore, in this scenario, the excluded volume of the hadrons $V_{\text {ex }}$ is chosen a bit smaller (cf. Tab. 4). In the region between $T=140 \mathrm{MeV}$ and $170 \mathrm{MeV}$ 


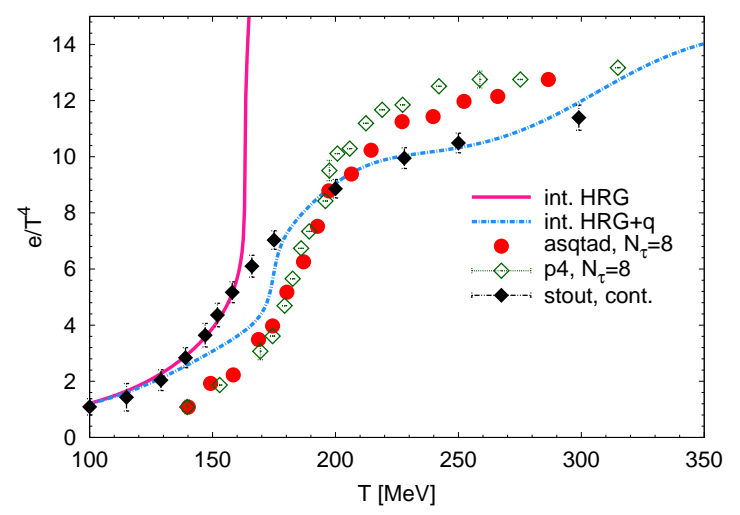

Figure 7. (Color online) Same quantity as above, again for the interacting HRG with (blue line) and without a quark phase (magenta line). The results are compared to lattice QCD data 78,81 85]. Up to $T_{c}$, the newer stout continuum extrapolated data (black points) is in reasonable agreement to the pure interacting HRG. Compared to the pure HRG, the scenario including quarks shows a suppression of $e / T^{4}$ in the region of $T_{c}$. This is caused by a slower increase of the energy due to the eigenvolume suppression of hadrons and cannot be compensated by the quarks which just start to populate the system at these temperatures. Therefore, the model with quarks lies in the region between newest stout action results and the somewhat older lattice data (asqtad and p4 action) which show large deviations from the HRG at $T<T_{c}$.

the interacting scenario shows a dip in the energy density. This is caused by the eigenvolume suppression of the hadrons and a rather slow increase of the quark abundances due to their coupling on the repulsive vector meson fields. At higher temperatures $(T>250 \mathrm{MeV})$, results from both model scenarios converge again, reflecting the fact that from this temperature on the system in both scenarios is dominated by quarks. Unlike in a Hagedorn resonance gas 101, given the large but still limited number of degrees of freedom in our model, thermodynamic quantities do not diverge.

Figure 7 shows the same quantity from the interacting HRG with and without quarks in comparison to lattice QCD results from Refs. 78, 81 85]. Up to $T_{c}$, the results from the purely hadronic model are in quantitatively good agreement with newest lattice data with the continuum extrapolated stout action [83 85]. Compared to the HRG, the somewhat older lattice data from the HotQCD collaboration [78, 81, 82] clearly underestimates the energy density in the hadronic regime $\left(T<T_{c}\right)$. In this figure, the impact of the quark phase and the associated excluded volume suppression on the thermodynamic properties of the model becomes obvious. As mentioned above, there is a dip in $e / T^{4}$ in the vicinity of $T_{c}$. In this region, the rather large eigenvolume of the hadrons leads to a flattening of the baryon density as a function of the temperature, while the quark density is only just starting to rise sharply and cannot compensate the lower hadron contribution (cf. dashed lines in Fig. 3). In the transition region, results from our model including quarks lie in the region between the lattice data from the HotQCD collaboration and newest continuum extrapolated Wuppertal-Budapest results. A convergence of more recent and highly anticipated data from the HotQCD collaboration, probably with physical pion masses and continuum extrapolation, towards our full-model results seems possible. 


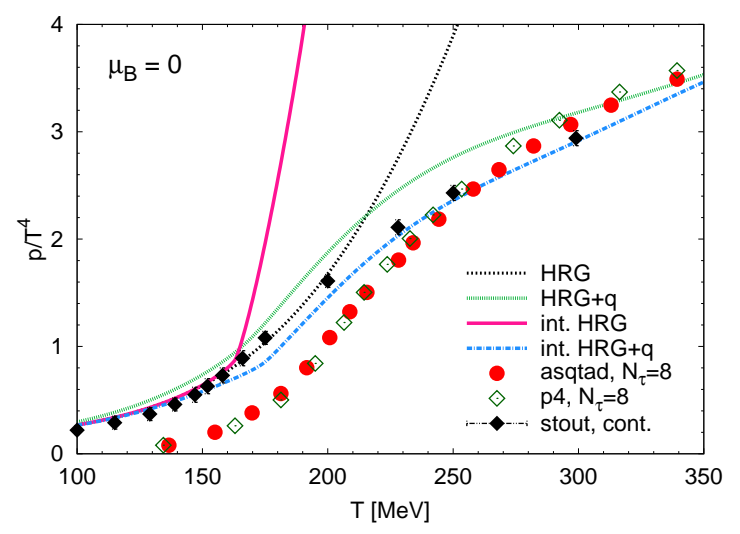

Figure 8. (Color online) Pressure over temperature to the fourth power from the chiral model in comparison to lattice QCD results at $\mu=0$. The line styles depicting the different model scenarios are as in Fig. 6] As for the energy, the lattice data show obvious discrepancies between the different lattice actions. The model with presumably correct degrees of freedom, the interacting HRG with the quark phase, shows reasonably good agreement to latest lattice data (stout, continuum extrapolated).

Figure 8 shows the pressure over the temperature to the fourth power as a function of $T$. Depicted are results from the presented model (with line styles as in the previous figures) compared to lattice data. Here, the same observations as for the energy can be made. The pressure in both scenarios without quarks rises monotonously and again much faster above $T_{c}$ in the interacting case due to the dropping effective masses. The scenarios including quarks differ again, mainly due to their difference in the coupling of particles to the vector fields. The pressure calculated with the interacting model reproduces the slope of the stout action results nicely, only undershooting lattice results in a small region around $T_{c}$. Again, compared to HRG results, older lattice data obviously yields too small values for $T<1.2 T_{c}$.

An important quantity, characterizing the phase transition, is the trace of the energy momentum tensor $e-3 p$, also known as interaction measure or trace anomaly, the basic thermodynamic quantity in lattice QCD from which other thermodynamic quantities can be obtained via integration. Figure 9 shows this quantity (again divided by $T^{4}$ ) as a function of the temperature calculated with the chiral model using the four different scenarios described above. As before, the results of the scenarios, which only include a hadronic phase without a volume suppression effect, show a rapid rise at $T_{c}$. The results of the other two scenarios, however, show a maximum slightly above $T_{c}$. Again, the ideal non-interacting scenario (dashed green line) shows a smoother transition between the hadronic phase and the quark phase. The fully interacting model (dashed blue line) exhibits a slowly increasing interaction measure below $T_{c}$, comparable to the dip in the energy density in this region, followed by a rapid rise due to dropping hadron masses and a slower decrease above the critical temperature.

Results from the latter two scenarios show a slope comparable to those from lattice QCD. Certainly, the chiral model does not reproduce the results of one lattice action consistently. In the vicinity of $T_{c}$, results from the ideal hadron and quark scenario (dashed green line) yield a qualitatively good agreement with lattice results using the continuum extrapolated stout action. With increasing temperatures $\left(T>T_{c}\right)$ 


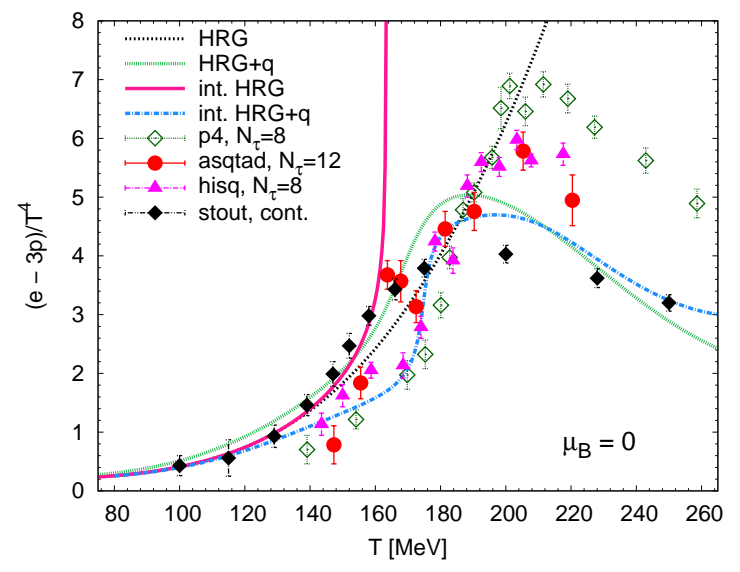

Figure 9. (Color online) Interaction measure, $(e-3 p) / T^{4}$, as a function of the temperature at $\mu_{B}=0$ in comparison to lattice data obtained with different fermion actions. Line styles and colors for the different scenarios as depicted in Fig. [6 additional lattice data taken from Ref. [79, 80]. Up to $T \approx T_{c}$ the data obtained with the continuum extrapolated stout action agrees well with the HRG with quarks scenario.

all lattice data show a smooth decline towards small values. This slope of the interaction measure is not reproduced with the chiral model, which, contrastingly, shows a rather flat behavior followed by a delayed decline at significantly higher temperatures $(T \sim 350 \mathrm{MeV})$. Since the applied Polyakov potential Eq. (18) has been fitted to reproduce lattice results in the pure gauge sector [63], this deviation in the slope must be attributed both, to the slowly decreasing meson fields in the presence of quarks and to hadrons occurring at temperatures well above $T_{c}$ (cf. Fig. 1 and 3). Additionally, at large temperatures, transverse gluons are known to contribute largely to the total pressure. However, the Polyakov loop corresponds exclusively to the longitudinal gauge field and, therefore, underestimates the pressure at very high T. In Ref. [46] K. Fukushima shows that the applicability of the Polyakov potential used in this work 63 breaks down above $T \sim 2 T_{c}$. This shortcoming could be another reason for the significant deviation of model results from lattice QCD in the high-temperature region.

A quantity which connects thermodynamic properties of the equation of state with the dynamics of heavy ion collisions is the velocity of sound $c_{s}$ which is deduced from thermodynamic quantities via

$$
c_{s}^{2}=\left.\frac{\partial p}{\partial \varepsilon}\right|_{s / \rho_{B}} .
$$

The square of the speed of sound of nuclear matter equals the change in pressure for a given change in energy along an isentropic expansion path. Thus, this important property controls the expansion dynamics of the whole fireball, also of sound-like fluctuations and density perturbations within the whole expanding system. The expansion dynamics and possible high density shock waves evolving from the collision of the two nuclei, in particular in uneven sized colliding nuclei, are likely to be reflected in final state particle observables [102 105]. Vice versa, the determination of the speed of sound from easily accessible particle observables may provide in-depth information 


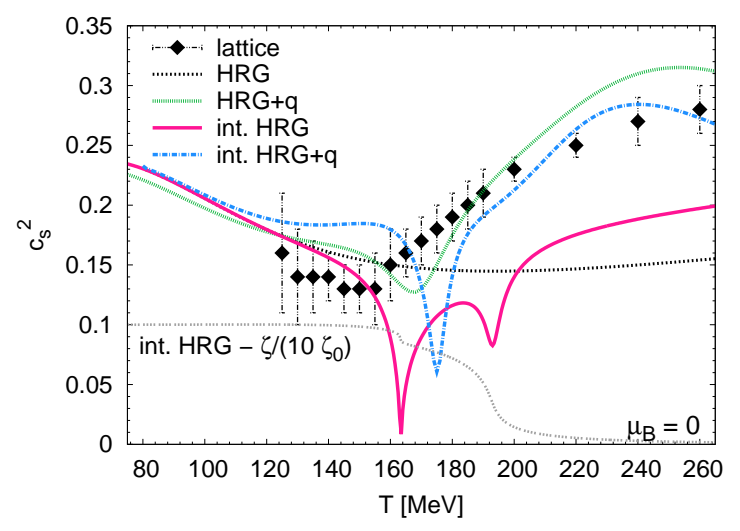

Figure 10. (Color online) Sound velocity squared at zero baryochemical potential for the different scenarios as described in the caption of Fig. [6 as a function of the temperature. For both, the pure HRG and the HRG with quarks, the resulting equation of state softens considerably if the interactions of the particles with the mesonic fields are taken into account. The second dip for the interacting HRG at $T \approx 190 \mathrm{MeV}$, which is also slightly visible in the interacting HRG with the quark phase, is caused by a sharp drop of the $\zeta$-field (dashed gray line). The lattice data (stout, continuum extrapolated) is almost flat up to $T_{c}$ and and rises with a slope comparable to the HRG+q scenarios for higher $T$ (taken from Ref. [113]).

on the thermodynamic properties of nuclear matter, especially in relation to the phase transition where $c_{s}$ significantly drops [106 112].

The velocity of sound squared as a function of the temperature calculated with the chiral model and from lattice QCD [113] is shown in Fig. 10, Again, the different parameter scenarios described above are considered and, as expected, it is observed that the smoother the transition of the thermodynamic quantities at $T_{c}$, the stiffer the equation of state. This seems plausible since a smooth change in effective degrees of freedom at the phase transition leads to much smoother course in energy density and pressure and thus in a much flatter derivative Eq. (26).

The interacting HRG (solid magenta line) shows the smallest $c_{s}^{2}$ at $T_{c}$ due to the rapid increase of degrees of freedom in a small range caused by the drop in $m^{*}$. When looking at the speed of sound for the two fully interacting scenarios, one also observes a second drop down to $c_{s}^{2}=0.1$ (or a small dip in $c_{s}^{2}$ if the quark phase is taken into account) at temperatures slightly above $T_{c}$. This local minimum is caused by the strange quark-antiquark condensate $\zeta$ which falls off at this point and affects $m^{*}$ as described in Eq. (3). For a better understanding, the $\zeta$-field $\left(0.1 \zeta(T) / \zeta_{0}\right)$ in the interacting HRG scenario is illustrated by the thin dashed gray line. The ideal HRG with the quark phase (green dashed line) shows only a small negative deviation from the ideal HRG (black dashed line) at $T_{c}$. As mentioned above, this is due to smooth changes in $p$ and $e$ at the phase transition (cf. Fig. 9). For higher temperatures the value quickly tends toward the ideal gas limit $c_{s}^{2}=1 / 3$, as expected.

In experiment, a distinctive softening of the EoS, i.e. a small $c_{s}^{2}$ in the transition region, strongly slows down the flow of matter during the collision process and thus stops a deflection of spectator matter. This, so-called, burning log effect [11, 115], causes an observable reduction of directed and elliptic flow of particles produced in semi-peripheral collisions, as was argued in Refs. [116 -120]. This behavior was experimentally observed by the NA49 collaboration Ref. [121]. At beam energies in 
the region between 30 and $40 \mathrm{~A} \mathrm{GeV}$, a collapse of the proton directed and elliptic flow was found. In addition, as studied in Refs. [122, 123], the softening of the equation of state in the transition region should be reflected in a strictly non-monotonic behavior of the HBT radii extracted from collisions in this intermediate energy region.

In contrast to these findings, there is no softening of the EoS in the lattice data. The sound velocity from Ref. [113] stays constant up to $T_{c}$ and rises for higher temperatures with a slope comparable to chiral model results with included quark phase. This, in fact, would rule out a significant delay in the expansion of the fireball and a prolonged lifetime of matter with temperature below $T_{c}$ as suggested in Refs. [114, 122].

Given these observations, we conclude that our EoS based on the interacting HRG including the quark phase, offers not only feasible degrees of freedom in a wide range of temperatures and densities but also exhibits properties consistent with experimental observations.

\section{Summary}

In this paper, results from a well tested chiral effective $\sigma-\omega$ model were presented, including all known hadronic degrees of freedom, which for this study was extended by a quark phase. The quarks were added in an PNJL-like approach in which they couple to an effective Polyakov loop potential. The chiral order parameter and the Polyakov loop order parameter for the deconfinement transition show a critical temperature $T \approx 165 \mathrm{MeV}$, which is in line with all latest lattice QCD predictions. It shows, that the chiral phase transition is mainly driven in the hadronic regime and there are no qualitative changes concerning the chiral transition when adding a quark phase.

At higher temperatures and densities hadronic degrees of freedom are effectively suppressed in favor of a purely quark-dominated QGP phase by eigenvolume corrections. Due to this fact, the model presented here offers a description of nuclear matter with the correct degrees of freedom in a wide range of temperature and baryochemical potential. Additionally, with the chiral model it is possible to study both, the chiral and the deconfinement phase transition. Fixing the value for the coupling strength of quarks to the repulsive meson vector fields, which has a major influence on the structure and the properties of the resulting phase diagram, reasonable nuclear ground state properties and a first-order liquid-gas phase transition can be reproduced. Nevertheless, the value of the nuclear matter compression modulus may restrict our choice of values for the finite particle volume and the repulsive quark vector couplings. For a future study, we intend to introduce a thermodynamically consistent implementation of temperature or density dependent eigenvolume corrections that could remedy this problem. For both, the chiral and the deconfinement phase transition, a smooth cross-over transition at vanishing and also finite baryochemical potentials is obtained, as previously shown without quarks for the chiral transition in Ref. [43].

The thermodynamic quantities from the chiral model, generally show a qualitatively reasonable agreement with lattice data, even though, in particular for the interaction measure there are still significant differences between the various lattice QCD results depending on the lattice action used. The softening of the sound velocity in the transition region was present in all scenarios except the pure HRG, where no rapid change of degrees of freedom takes places. For the HRG with the included quark phase the largest drop in $c_{s}^{2}$ was observed. If all particles in the model fully couple to 
the mesonic fields, the sound velocity squared gets close to zero at the phase transition. This significant softening meets a major constraint on the EoS as it is suggested by experimental observations on the drop in the flow by the NA49 collaboration [121].

The chiral effective model presented here, is able to provide an EoS to be used in hydrodynamic models for studying the dynamics of heavy ion collisions. This is a major benefit of an integrated model which has the right degrees of freedom in a wide range of the phase diagram. Consequently, the resulting EoS is well suited for calculations with hybrid models [104, 124 128], like the UrQMD hybrid model [129], since it exhibits known features of QCD and has exactly the same degrees of freedom in the hydrodynamic stage as in the early and subsequent cascade stage.

\section{Acknowledgements}

This work was supported by BMBF, GSI, and by the Hessian excellence initiative LOEWE (Landesoffensive zur Entwicklung Wissenschaftlich-ökonomischer Exzellenz) through the Helmholtz International Center for FAIR (HIC for FAIR), and the Helmholtz Graduate School for Hadron and Ion Research (HGS-HIRe). Computational resources were provided by the Center for the Scientific Computing (CSC) of the Goethe University Frankfurt. J. S. acknowledges a Feodor Lynen fellowship of the Alexander von Humboldt foundation. The authors thank M. Bleicher for fruitful discussion.

\section{References}

[1] Arsene I et al. (BRAHMS) 2005 Nucl. Phys. A757 1-27

[2] Back B B et al. 2005 Nucl. Phys. A757 28-101

[3] Adams J et al. (STAR) 2005 Nucl. Phys. A757 102-183

[4] Adcox K et al. (PHENIX) 2005 Nucl. Phys. A757 184-283

[5] Nambu Y and Jona-Lasinio G 1961 Phys. Rev. 122 345-358

[6] Kirzhnits D A and Linde A D 1972 Phys. Lett. B42 471-474

[7] Weinberg S 1974 Phys. Rev. D9 3357-3378

[8] Theis J, Graebner G, Buchwald G, Maruhn J, Greiner W et al. 1983 Phys.Rev. D28 2286-2290

[9] Karsch F 2000 Nucl. Phys. Proc. Suppl. 83 14-23

[10] Fodor Z and Katz S D 2002 Phys. Lett. B534 87-92

[11] Fodor Z, Katz S D and Szabo K K 2003 Phys. Lett. B568 73-77

[12] Fodor Z and Katz S D 2004 JHEP 04050

[13] Karsch F 2005 J. Phys. G31 S633-S640

[14] Aoki Y, Endrodi G, Fodor Z, Katz S D and Szabo K K 2006 Nature 443 675-678

[15] Cheng M et al. 2006 Phys. Rev. D74 054507

[16] Cheng M et al. 2007 Phys. Rev. D75 034506

[17] DeTar C 2008 PoS LAT2008 001

[18] Petreczky P 2009 Nucl. Phys. A830 11c-18c

[19] Fodor Z and Katz S D 2002 JHEP 03014 
[20] Fodor Z, Katz S D and Schmidt C 2007 JHEP 03121

[21] Allton C, Ejiri S, Hands S, Kaczmarek O, Karsch F et al. 2002 Phys.Rev. D66 074507

[22] de Forcrand P and Philipsen O 2003 Nucl. Phys. B673 170-186

[23] D'Elia M and Lombardo M P 2003 Phys. Rev. D67 014505

[24] D'Elia M, Di Renzo F and Lombardo M P 2007 Phys. Rev. D76 114509

[25] Allton C R et al. 2005 Phys. Rev. D71 054508

[26] de Forcrand P and Philipsen O 2008 JHEP 11012

[27] Kaczmarek O et al. 2011 Phys. Rev. D83 014504

[28] Wu L K, Luo X Q and Chen H S 2007 Phys. Rev. D76 034505

[29] Stephanov M A, Rajagopal K and Shuryak E V 1998 Phys. Rev. Lett. 81 48164819

[30] Stephanov M A, Rajagopal K and Shuryak E V 1999 Phys. Rev. D60 114028

[31] Stephanov M A 2006 PoS LAT2006 024

[32] Endrodi G, Fodor Z, Katz S and Szabo K 2011 JHEP 1104001

[33] Gyulassy M and McLerran L 2005 Nucl. Phys. A750 30-63

[34] Walecka J D 1974 Annals Phys. 83 491-529

[35] Serot B D and Walecka J D 1986 Adv.Nucl.Phys. 16 1-327

[36] Boguta J and Bodmer A R 1977 Nucl. Phys. A292 413-428

[37] Boguta J and Stöcker H 1983 Phys.Lett. B120 289-293

[38] Khvorostukhin A S, Toneev V D and Voskresensky D N 2007 Nucl. Phys. A791 180-221

[39] Khvorostukhin A S, Toneev V D and Voskresensky D N 2008 Nucl. Phys. A813 313-346

[40] Nambu Y and Jona-Lasinio G 1961 Phys. Rev. 124 246-254

[41] Papazoglou P, Schramm S, Schaffner-Bielich J, Stöcker H and Greiner W 1998 Phys.Rev. C57 2576-2588

[42] Papazoglou P, Zschiesche D, Schramm S, Schaffner-Bielich J, Stöcker H et al. 1999 Phys.Rev. C59 411-427

[43] Rau P, Steinheimer J, Schramm S and Stöcker H 2012 Phys.Rev. C85 025204

[44] Rau P, Steinheimer J, Schramm S and Stöcker H 2012 Cent. Eur. J. Phys. 10(6) 1302-1305

[45] Fukushima K 2004 Phys.Lett. B591 277-284

[46] Fukushima K 2008 Phys.Rev. D77 114028

[47] Ratti C, Thaler M A and Weise W 2006 Phys.Rev. D73 014019

[48] Roessner S, Ratti C and Weise W 2007 Phys.Rev. D75 034007

[49] Schaefer B J, Pawlowski J M and Wambach J 2007 Phys.Rev. D76 074023

[50] Sasaki C, Friman B and Redlich K 2007 Phys.Rev. D75 074013

[51] Megias E, Ruiz Arriola E and Salcedo L 2006 Phys.Rev. D74 065005

[52] Ghosh S K, Mukherjee T K, Mustafa M G and Ray R 2006 Phys.Rev. D73 114007 
[53] Meisinger P N and Ogilvie M C 1996 Phys.Lett. B379 163-168

[54] Steinheimer J, Schramm S and Stöcker H 2009 (Preprint 0909.4421)

[55] Steinheimer J, Schramm S and Stöcker H 2011 J.Phys.G G38 035001

[56] Dexheimer V and Schramm S 2008 Astrophys.J. 683 943-948

[57] Serot B D and Walecka J D 1997 Int.J.Mod.Phys. E6 515-631

[58] Beringer J et al. (Particle Data Group) 2012 Phys.Rev. D86 010001

[59] Nakamura K et al. (Particle Data Group) 2010 J. Phys. G37 075021

[60] Dexheimer V and Schramm S 2010 Phys.Rev. C81 045201

[61] Meisinger P N and Ogilvie M C 1996 Nucl.Phys.Proc.Suppl. 47 519-522

[62] Ratti C, Thaler M A and Weise W 2006 (Preprint nucl-th/0604025)

[63] Ratti C, Roessner S, Thaler M and Weise W 2007 Eur.Phys.J. C49 213-217

[64] Boyd G, Engels J, Karsch F, Laermann E, Legeland C et al. 1996 Nucl.Phys. B469 419-444

[65] Meisinger P N, Ogilvie M C and Miller T R 2004 Phys.Lett. B585 149-154

[66] Steinheimer J and Schramm S 2011 Phys. Lett. B696 257-261

[67] Rischke D H, Gorenstein M I, Stöcker H and Greiner W 1991 Z. Phys. C51 485-490

[68] Mishra M and Singh C 2007 Phys.Rev. C76 024908

[69] Cleymans J, Gorenstein M I, Stalnacke J and Suhonen E 1993 Phys.Scripta 48 $277-280$

[70] Ma B Q, Zhang Q R, Rischke D and Greiner W 1993 Phys.Lett. B315 29-33

[71] Yen G D, Gorenstein M I, Greiner W and Yang S N 1997 Phys.Rev. C56 22102218

[72] Gorenstein M I, Kostyuk A and Krivenko Y 1999 J.Phys. G25 L75-L83

[73] Bugaev K, Gorenstein M I, Stöcker H and Greiner W 2000 Phys.Lett. B485 $121-125$

[74] Baacke J 1977 Acta Phys. Polon. B8 625

[75] Hagedorn R and Rafelski J 1980 Phys. Lett. B97 136

[76] Gorenstein M I, Petrov V K and Zinovev G M 1981 Phys. Lett. B106 327-330

[77] Hagedorn R 1983 Z. Phys. C17 265

[78] Bazavov A et al. 2009 Phys. Rev. D80 014504

[79] Bazavov A and Petreczky P (HotQCD) 2010 J. Phys. Conf. Ser. 230012014

[80] Bazavov A and Petreczky P (HotQCD) 2011 Phys.Part.Nucl.Lett. 8 860-864

[81] Cheng M, Hendge P, Jung C, Karsch F, Kaczmarek O et al. 2009 Phys. Rev. D79 074505

[82] Cheng M et al. 2010 Phys. Rev. D81 054504

[83] Borsanyi S et al. (Wuppertal-Budapest) 2010 JHEP 1009073

[84] Borsanyi S et al. 2010 JHEP 11077

[85] Aoki Y, Borsanyi S, Durr S, Fodor Z, Katz S D et al. 2009 JHEP 0906088

[86] Bazavov A, Bhattacharya T, Cheng M, DeTar C, Ding H et al. 2012 Phys.Rev. D85 054503 
[87] Mohr P, Taylor B and Newell D 2011 CODATA Database http://physics.nist.gov/constants

[88] Steinheimer J, Schramm S and Stöcker H 2011 Phys. Rev. C84 045208

[89] Gerber P and Leutwyler H 1989 Nucl. Phys. B321 387

[90] Mishra A, Schramm S and Greiner W 2008 Phys. Rev. C78 024901

[91] Markert C, Bellwied R and Vitev I 2008 Phys.Lett. B669 92-97

[92] Bellwied R and Markert C 2010 Phys.Lett. B691 208-213

[93] Ratti C, Bellwied R, Cristoforetti M and Barbaro M 2012 Phys.Rev. D85 014004

[94] Waldhauser B, Theis J, Maruhn J, Stöcker H and Greiner W 1987 Phys.Rev. C36 1019-1026

[95] Mocsy A, Mishustin I N and Ellis P J 2004 Phys. Rev. C70 015204

[96] Bowman E S and Kapusta J I 2009 Phys. Rev. C79 015202

[97] Blaizot J 1980 Phys.Rept. 64171

[98] Shlomo S, Kolomietz V and Colò G 2006 Eur. Phys. J. A30(1) 23-30

[99] Kostyuk A, Gorenstein M, Stöcker H and Greiner W 2001 Phys.Rev. C63 044901

[100] Bugaev K A 2008 Nucl.Phys. A807 251-268

[101] Hagedorn R 1968 Nuovo Cim.Suppl. 6 311-354

[102] Rischke D H, Pursun Y and Maruhn J A 1995 Nucl.Phys. A595 383-408

[103] Dumitru A and Rischke D 1999 Phys.Rev. C59 354-363

[104] Bass S and Dumitru A 2000 Phys.Rev. C61 064909

[105] Knoll J, Randrup J, Fuchs C, Aichelin J, Bleicher M et al. 2011 Lect.Notes Phys. $814531-680$

[106] Scheid W, Muller H and Greiner W 1974 Phys.Rev.Lett. 32 741-745

[107] Mohanty B and Alam J e 2003 Phys.Rev. C68 064903

[108] Bravina L, Arsene I, Nilsson M, Tywoniuk K, Zabrodin E et al. 2008 Phys.Rev. C78 014907

[109] Castorina P, Cleymans J, Miller D and Satz H 2010 Eur.Phys.J. C66 207-213

[110] Rau P, Steinheimer J, Betz B, Petersen H, Bleicher M et al. 2010 (Preprint 1003.1232)

[111] Senger P, Bratkovskaya E, Andronic A, Averbeck R, Bellwied R et al. 2011 Lect.Notes Phys. 814 681-847

[112] Steinheimer J and Bleicher M 2012 Eur.Phys.J. A48 100

[113] Borsanyi S, Endrodi G, Fodor Z, Katz S, Krieg S et al. 2012 JHEP 1208053

[114] Rischke D H and Gyulassy M 1996 Nucl.Phys. A597 701-726

[115] Hung C and Shuryak E V 1998 Phys.Rev. C57 1891-1906

[116] Soff S, Bass S A, Bleicher M, Stöcker H and Greiner W 1999 (Preprint nucl-th/9903061)

[117] Li B A, Ko C, Sustich A T and Zhang B 1999 Phys.Rev. C60 011901

[118] Zheng Y M, Ko C, Li B A and Zhang B 1999 Phys.Rev.Lett. 83 2534-2536

[119] Stöcker H 2005 Nucl.Phys. A750 121-147

[120] Li Q, Li Z, Soff S, Bleicher M and Stöcker H 2006 J.Phys. G32 151-164 
[121] Alt C et al. (NA49) 2003 Phys.Rev. C68 034903

[122] Rischke D H and Gyulassy M 1996 Nucl.Phys. A608 479-512

[123] Li Q, Steinheimer J, Petersen H, Bleicher M and Stöcker H 2009 Phys.Lett. B674 111-116

[124] Teaney D, Lauret J and Shuryak E 2001 (Preprint nucl-th/0110037)

[125] Hirano T, Heinz U W, Kharzeev D, Lacey R and Nara Y 2006 Phys.Lett. B636 299-304

[126] Nonaka C and Bass S A 2007 Phys.Rev. C75 014902

[127] Song H, Bass S A, Heinz U, Hirano T and Shen C 2010 Phys.Rev.Lett. 106 192301

[128] Yan Y L, Cheng Y, Zhou D M, Dong B G, Cai X et al. 2011 (Preprint 1110.6704)

[129] Petersen, H and Steinheimer, J and Burau, G and Bleicher, M and Stöcker, H 2008 Phys.Rev. C78 044901 